\title{
Math-Net.Ru
}

Общероссийский математический портал

И. В. Протасов, Уравнения в $\beta G$ и разложимость абелевых групп, Матем. заметки, 1999, том 66, выпуск 6, 951-953

DOI: https://doi.org/10.4213/mzm1241 
Использование Общероссийского математического портала Math-Net.Ru подразумевает, что вы прочитали и согласны с пользовательским соглашением http://www. mathnet.ru/rus/agreement

Параметры загрузки:

IP : 54.209 .52 .79

26 апреля 2023 г., 04:16:31

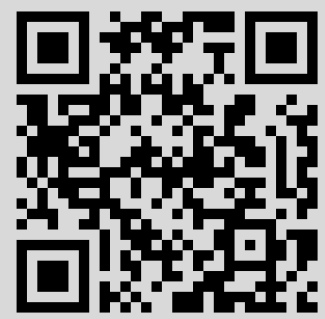




\section{УРАВНЕНИЯ В $\beta G$ И РАЗЛОЖИМОСТЬ АБЕЛЕВЫХ ГРУПП}

\section{И.В. Протасов}

Топологическое пространство $X$ называется разложимым ( $\omega$-разложсимым), если $X$ можно разбить на два (счетное число) плотных подмножества. Топологическая группа $G$ называется разложимой ( $\omega$-разложимой), если ее пространство разложимо ( $\omega$-разложимо). Все рассматриваемые группы предполагаются абелевыми, а топологии - хаусдорфовьми.

Комфорт и ван Милл доказали [1] разложимость недискретной топологической группы $G$ с конечной подгруппой $B(G)=\{g \in G: 2 g=0\}$. Эта теорема усилена в [2]: недискретная топологическая группа $G$ с конечной подгруппой $B(G) \omega$-разложима. Примеры недискретных неразложимых топологических групп построены в [3]-[5] при дополнительных к системе аксиом $\mathbf{Z F C}$ теоретико-множественных предположениях. В [6] доказано существование моделей ZF C, в которых любая недискретная топологическая группа $\omega$-разложима. Таким образом, неразложимость - весьма экзотическое свойство топологических групп. Подробнее о разложимости топологических групп см. обзоры [7], [8].

C другой стороны, на любой бесконечной группе $G$ можно определить в $\mathbf{Z F C}$ недискретную неразложимую (и даже максимальную) топологию, в которой непрерывны все сдвиги $x \mapsto x+g$, $g \in G$. Топологическое пространство $X$ без изолированных точек называется максимальным, если $X$ имеет изолированные точки в любой более сильной топологии. О максимальных топологиях на группах см. статью [9]. 
В чем же причина разложимости топологических групп? Цель заметки - доказательство следующей теоремы, которая существенно проясняет этот вопрос.

Теорема. Пусть $\tau$-недискретная топология на группе $G$, в которой непрерывны все сдвиги $x \mapsto x+g, g \in G$. Предположим, что для некоторого челого числа $m$ отображение $x \mapsto m x$ непрерывно и подгруппа $\{g \in G: m(m-1) g=0\}$ конечна. Если $|m|>1$, mо $(G, \tau)$ ш-разложима. Если $m=-1$, то $(G, \tau)$ разложима.

Таким образом, причина разложимости топологической группы кроется не в совместной непрерьвности операции сложения, а в непрерьвности сдвигов и отображения $x \mapsto-x$.

Доказательство теоремы разобьем на несколько фрагментов, сопровождая каждый из них необходимьми определениями и ссылками. Отметим, что решающую роль в этом доказательстве играют некоторые уравнения в чех-стоуновой компактификации группы.

1) Для произвольной группы $G$ обозначим через $\beta G$ чех-стоунову компактификацию группы $G$ как дискретного пространства. Элементами компактного пространства $\beta G$ являются всевозможные ультрафильтры на группе $G$, а базу топологии образуют множества $\bar{A}=\{p \in \beta G: A \in p\}$, где $A$ пробегает все подмножества группы $G$. Отождествим группу $G$ с подмножеством всех главных ультрафильтров из $\beta G$, а подмножество $\beta G \backslash G$ всех свободных ультрафильтров обозначим через $G^{*}$.

Операция сложения элементов группы $G$ естественно продолжается на $\beta G$. Сумму ультрафильтров $p, q \in \beta G$ определим указанием всех подмножеств $A \subseteq G$, которые являются элементами уль трафиильтра $p+q$ :

$$
A \in p+q \Longleftrightarrow\{g \in G: A-g \in q\} \in p .
$$

Операция сложения ультрафильтров ассоциативна, непрерывна по первому аргументу при фиксированном втором аргументе и непрерьвна по второму аргументу, если фиксированньй первый аргумент является главным ультраффильтром. Подробную информацию о строении полугруппы $\beta G$, а также различные ее приложения можно найти в [10], [11].

Для любого целого числа $m$ отображение $x \mapsto m x$ также естественно продолжается на $\beta G$. Для ультрафильтра $p \in \beta G$ обозначим через $m p$ ультрафильтр с базой $\{m A: A \in p\}$.

Нам понадобится следующее утверждение об уравнениях в $\beta G$.

Tеорема А. Пусть $G$ - бесконечная группа, $m-$ челое число, $m \neq 0,1$. Eсли подгруппа $\{g \in G: m(m-1) g=0\}$ конечна, то уравнение $x+p=y+m p$ неразрешимо в $\beta G$ для любого ультрафильтра $p \in G^{*}$.

Для $m=2$ это утверждение доказано в [12], а для $G=\mathbb{Z}$ и $m \neq 0,1$ - в [13]. Доказательство теоремы А не требует новых идей и проводится по схеме, изложенной в $[12, \S 2]$.

2) Пусть $\tau$ - топология на группе $G$ такая, что все отображения $x \mapsto x+g, g \in G$, непрерьвны. Обозначим через $\bar{\tau}$ полугруппу всех ультрафильтров на $G$, сходящихся к 0 в топологии $\tau$.

Теорема Б (см. [6, теорема 1.9]). Если для заданного натурального числа $n$ топологическое пространство $(G, \tau)$ нельзя разбить на $n$ плотных подмножсеств, то каждый минимальный правый идеал полугруппы $\bar{\tau}$ содержит не более чем $n-1$ элементов.

3) ТЕОРЕМА ВАН ДАУЭНА-ИЛЬЯНЕСА (см. [14]). Если для любого натурального иисла $n$ топологическое пространство $X$ можно разбить на $n$ плотных подмножеств, то $X$ $\omega-$ разлохимов.

4) Пусть $(G, \tau)$ удовлетворяет условиям теоремы и $|m|>1$. Ввиду п. 2 и п. 3 достаточно показать, что каждый минимальный правый идеал полугруппы $\bar{\tau}$ бесконечен. Допустим противное и зафиксируем конечный минимальный правый идеал $M$ полугруппы $\bar{\tau}$. Так как топология $\tau$ недискретна, то $M$ содержит некоторый свободный ультрафильтр $q$. Поскольку отображение $x \mapsto m x$ непрерьвно в топологии $\tau$, то $m^{k} q \in \bar{\tau}$ для любого натурального числа $k$. Следовательно, $q+m^{k} q \in M$. Так как правый идеал $M$ конечен и $|m|>1$, то найдутся натуральные числа $r, s$ такие, что $r>s$ и $q+m^{r} q=q+m^{s} q$. Положим $p=m^{s} q$. Тогда $q+m^{r-s} p=q+p$, что противоречит п. 1 .

Пусть $m=-1$, однако группа $(G, \tau)$ неразложима. Ввиду п. 1 каждый минимальный правьй идеал полугруппы $\bar{\tau}$ одноэлементен. Пусть $\{q\}$ - минимальный правый идеал полугруппы $\bar{\tau}$. Так как отображение $x \mapsto-x$ непрерывно в топологии $\tau$, то $-q \in \bar{\tau}$. Значит, $q-q=q$, что противоречит п. 1. 
ЗАмечАниЕ. Покажем, что условие конечности подгруппы $\{g \in G: m(m-1) g=0\}$ в доказанной теореме существенно. Допустим, что эта подгруппа бесконечна. Тогда бесконечна, по крайней мере, одна из подгрупп $G_{m}=\{g \in G: m g=0\}$ либо $G_{m-1}=\{g \in G:(m-1) g=0\}$. Пользуясь теоремой 1.2 из [9], снабдим одну из подгрупп $G_{m}, G_{m-1}$ максимальной и, следовательно, неразложимой топологией $\tau^{\prime}$, в которой непрерьвны сдвиги. Продолжим $\tau^{\prime}$ до топологии $\tau$ на группе $G$, в которой непрерывны сдвиги. В первом случае $m x=0$ для любого элемента $x$ из открытой в $\tau$ подгруппы $G_{m}$. Во втором случае $m x=x$ для любого элемента $x$ из открытой в $\tau$ подгруппы $G_{m-1}$. Значит, отображение $x \mapsto m x$ непрерьвно в топологии $\tau$.

ВоПрос 1 . Пусть $G$ - бесконечная группа, $m$ - целое число, $|m|>1$. Допустим, что подгруппа $\{g \in G: m(m-1) g=0\}$ конечна. Можно ли группу $G$ разбить на счетное число подмножеств, плотных в любой топологии с непрерьвными сдвигами и непрерывным отображением $x \mapsto m x$ ? Подчеркнем, что разбиение не должно зависеть от выбора топологии. Для свободной группы произвольной мощности такое разбиение можно построить, модифицируя конструкцию из [15].

ВопРос 2. Пусть $G$ - бесконечная группа с конечной подгруппой $\{g \in G: 2 g=0\}$. Верно ли, что $(G, \tau) \omega$-разложима, если $\tau$ - недискретная топология на $G$, в которой непрерывны все сдвиги и отображение $x \mapsto-x$ ?

\section{СПИСОК ЦИТИРОВАННОЙ ЛИТЕРАТУРЫ}

1. Comfort W. W., van Mill J. // Proc. Amer. Math. Soc. 1994. V. 120. № 3. P. 687-696. 2. Протасов И. В. // Укр. матем. ж. 1997. Т. 49. №10. С. 1385-1395. 3. Малыхин В.И. // Докл. АН СССР. 1975. Т. 220. №1. С. 27-30. 4. Зеленюк Е. Г. // Матем. студ. 1996. Т. 6. С. 41-52. 5. Зеленюк Е. Г., Протасов И. В. // Докл. НАН Украины. 1997. № 3. С. 7-11. 6. Протасов И.В. // Укр. матем. ж. 1998. Т. 50. №12. С. 1646-1655. 7. Протасов И. В. // Матем. студ. 1998. Т. 9. №2. С. 130-148. 8. Comfort W. W. // Topology Appl. 1996. V. 74. Р. 149-167. 9. Протасов И. В. // Сиб. матем. ж. 1998. Т. 39. №6. С. 1368-1381. 10. Hindman N., Strauss D. Algebra in the Stone-Čech Compactification. Berlin-New York: de Gruyter, 1998. 11. Protasov I. V. Combinatorics of Numbers. Math. Stud. Monogr. Ser. V. 2, 1997. 12. Протасов И. В. // Матем. сб. 1996. Т. 187. № 2. С. 131-140. 13. Протасов И. В. // Укр. матем. ж. 1997. Т. 49. № 4. С. 563-570. 14. Illanes A. // Proc. Amer. Math. Soc. 1996. V. 124. № 4. Р. 1243-1246. 15. Протасов И. В., Юрчук Т. Л . // Докл. НАН Украины. 1997. № 8. С. 38-40. 